Editorial Office Notes:

RES-16-230.R2

ORIGINAL ARTICLE

Received 25 March 2016

Invited to revise 25 May and 20 July 2016

Revised 26 June and 4 August 2016

Accepted 4 August 2016

Associate Editor: Lonny Yarmus

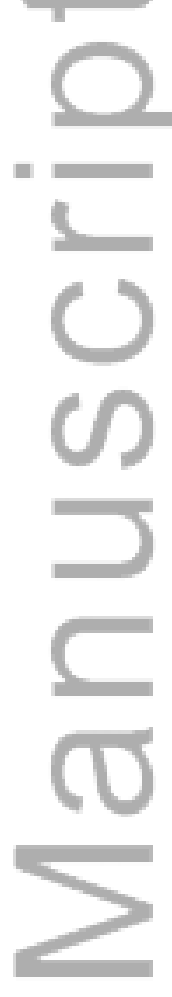

1

$\longrightarrow$

1
+1

(5)

2

This is the author manuscript accepted for publication and has undergone full peer review but has not been through the copyediting, typesetting, pagination and proofreading process, which may lead to differences between this version and the Version of Record. Please cite this article as doi: $10.1111 /$ resp. 12932

This article is protected by copyright. All rights reserved. 


\section{Accuracy and Safety of Ward Based Pleural Ultrasound in the Australian}

\section{Healthcare System}

Gary Hammerschlag, Matthew Denton, Peter Wallbridge, Louis Irving and Daniel Steinfort

Department of Respiratory and Sleep Medicine. The Royal Melbourne Hospital,

Grattan Street, Parkville, VIC, AUSTRALIA

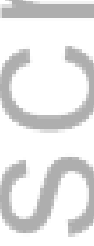

Correspondence:

Gary Hammerschlag, MBBS, FRACP.

Department of Respiratory and Sleep Medicine. The Royal Melbourne Hospital,

Grattan Street, Parkville, VIC, AUSTRALIA 3050.

Email: gary.hammerschlag@mh.org.au

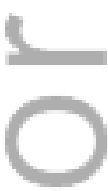

\section{Summary at a Glance}

Thoracic physician performed ultrasound has been shown to be safe and effective.We perform a retrospective review of pleural procedures performed at our institution with the aid of thoracic physician operated ultrasound, and we show that in the Australian public health system, it remains safe and effective 


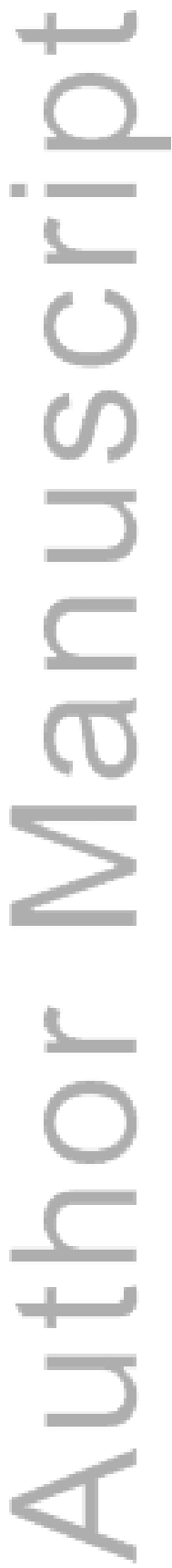

This article is protected by copyright. All rights reserved. 


\section{ABSTRACT}

BACKGROUND AND OBJECTIVE: Ultrasound has been shown to improve the accuracy and safety of pleural procedures. Studies to date have been performed in large, specialised units, where pleural procedures are performed by a small number of highly specialised physicians. There are no studies examining the safety and accuracy of ultrasound in the Australian healthcare system where procedures are performed by junior doctors with a high staff turnover.

METHODS: We performed a retrospective review of the ultrasound database in the Respiratory Department at the Royal Melbourne Hospital to determine accuracy and complications associated pleural procedures.

RESULTS: 357 ultrasounds were performed between October 2010 and June 2013. Accuracy of pleural procedures was 350/356 (98.3\%). Aspiration of pleural fluid was successful in 121/126 (96\%) of cases. Two (0.9\%) patients required chest tube insertion for management of pneumothorax. There were no recorded pleural infections, haemorrhage or viscera puncture.

CONCLUSION: Ward based ultrasound for pleural procedures is safe and accurate when performed by appropriately trained and supported junior medical officers. Our findings support this model of pleural service care in the Australian Healthcare system.

Keywords: Diagnostic Imaging, Pleural effusion, Safety, Thoracentesis,

Ultrasonography

Short Title: Ward-Based Pleural Ultrasound 


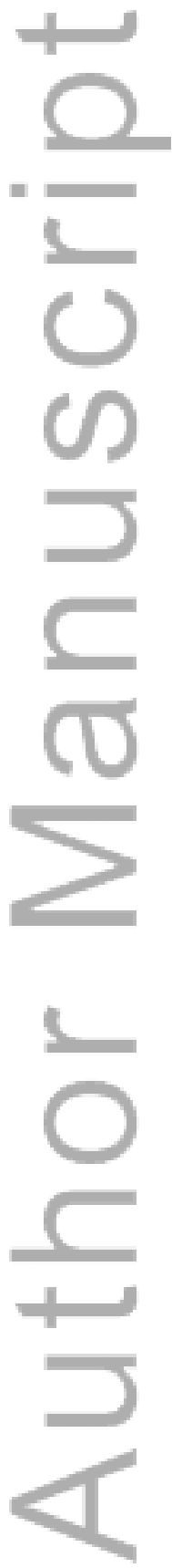

This article is protected by copyright. All rights reserved. 


\section{INTRODUCTION}

Ultrasound has high sensitivity and specificity for the detection of pleural effusions, being superior to chest radiography and equal to CT for this purpose ${ }^{1}$. Ultrasound has a number of further advantages above conventional imaging, being portable, low-cost and radiation free. Consequently, there have been mounting calls for adoption of this technique into daily practice ${ }^{2}$. While imaging is considered essential for confirming the presence of an effusion, often an invasive pleural procedure is required for diagnosis of the underlying cause. Thoracic ultrasound has been shown to both increase diagnostic yield and reduce complications of thoracocentesis ${ }^{3}$, and thus is now recommended for all invasive pleural procedures ${ }^{4}$.

Thoracic ultrasound is increasingly being utilised by respiratory physicians for the assessment and management of pleural effusions ${ }^{5}$. While there is a growing body of evidence to suggest that this practice is acceptably accurate, safe and cost effective ${ }^{6-8}$, the majority of these studies were conducted by highly experienced consultant respiratory physicians in dedicated pleural units. As few hospitals in Australia are large enough to support a dedicated pleural unit, the generalisability of these findings to smaller, less-specialised hospitals with relatively low patient numbers and high clinician turnover remains unclear.

This article is protected by copyright. All rights reserved. 
The Department of Respiratory Medicine at the Royal Melbourne Hospital (RMH), Parkville, Australia, offers a thoracic ultrasound service for the evaluation and management of pleural disease. Ultrasound assessments are performed by large number of clinicians with varying levels of experience, who are accredited in the use of thoracic ultrasound. Supervising clinicians had completed a Certificate of Clinician Performed Ultrasound through the Australasian Society of Ultrasound in Medicine (ASUM). Respiratory registrars perform the majority of ultrasound assessments with senior clinicians available for support.

\section{METHODS}

This retrospective audit was adapted from the protocol previously defined by Rahman

${ }^{6}$. Ethics approval was granted by the RMH Department of Research Integrity and Ethics (QA2014210) prior to commencement of the study.

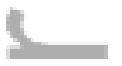

The study was conducted at the RMH, a tertiary hospital in Parkville, Australia. The ultrasound service run by the Department of Respiratory Medicine has maintained a database of all thoracic ultrasound assessments performed between October 2010 and June 2013. Details recorded included the date of the assessment, patient identification number, primary operator, indication (where stated), ultrasound findings, procedure (if performed), pathology results and any complications recorded at the time. 
Pathology and radiology records were crosschecked to obtain any missing data and ensure correctness of the information within the database.

Ultrasound assessments during this time were performed using a SonoSite M-Turbo and Sonosite Edge (SonoSite, Inc USA) with a 2-6 Hz curvilinear abdominal probe. All ultrasound assessments performed over this period for the investigation of pleural effusions, or those in which a pleural effusion was noted during the assessment, were reviewed by a single un-blinded assessor. Indication for assessment by the ultrasound service was considered either diagnostic (confirming the presence or absence of an effusion) or interventional (pleural aspiration, insertion of an intercostal catheter or both). Instances where both a diagnostic and therapeutic procedure was performed were considered separately. Diagnostic ultrasound assessments were performed if the effusion was found to be absent or insufficient for drainage, if intervention was not clinically indicated, or if no safe insertion site could be identified.

Table 1 lists the criteria used to determine the diagnostic accuracy of ultrasound assessments.

Table 2 outlines the criteria used to determine the safety of pleural procedures to obtain pleural fluid. Relevant information was collected for all patients from the ultrasound service database and crosschecked with both pathology and radiology records to determine the presence of post-procedural complications. Post procedural radiology was reviewed for a pneumothorax or haemothorax, and pathology was 
reviewed for increased inflammatory markers and rise in white cell count suggesting an infection. In a small number of cases appraisal of medical records was required to evaluate for potential adverse outcomes.

Finally, the number of cases in which assistance was requested from either radiology or cardiothoracic surgery following ultrasound assessment were recorded. Input was sought in cases where the operator required assistance with diagnostic interpretation of scans, as well as for interventional scans when the operator was unable to successfully obtain pleural fluid, despite detecting the presence of an effusion.

The $95 \%$ confidence interval for accuracy was calculated using the modified Wald method for binary data.

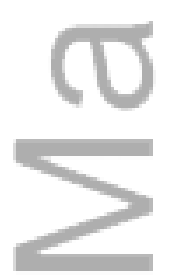

\section{RESULTS}

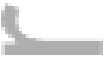

\section{Utilisation of Ultrasound Service}

Over a period of 2 years and 8 months, 357 scans were performed on 314 patients (mean age 66 years, $36 \%$ female). Over this period, 17 primary operators were involved in the thoracic ultrasound service, including 9 Respiratory Registrars who conducted 288/357 (80.7\%) ultrasound assessments. An effusion was demonstrated in 
$344 / 357$ (96.4\%) of ultrasound assessments. In 13/357 (3.6\%) no effusion was demonstrated, and in one instance a hydro-pneumothorax was diagnosed.

Diagnostic ultrasound was performed in $122(34.2 \%)$. Interventional scans occurred in $235 / 357(65.8 \%)$ of cases, with $126 / 357$ (35.3\%\%) undergoing pleural aspiration. Aspiration was successful in 121/126 (96\%). Intercostal drain insertion occurred in $103 / 357$ (28.9\%), and 6/357 (1.7\%) patients had both interventions performed.

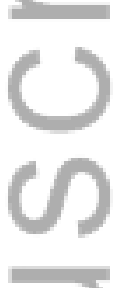

\section{Diagnostic Accuracy}

Data pertaining to diagnostic accuracy of respiratory physician-delivered ultrasound scans were available in all but one case $(356 / 357,99.7 \%)$. In this case, no ultrasound images were recorded to allow for assessment of accuracy and thus it was omitted from analysis. In patients undergoing thoracentesis, pleural fluid was successfully obtained in 230/235 (98\%) of cases (figure 1).

In 110/357 (30.1\%) scans ultrasound findings were confirmed by concurrent radiology (CT, formal radiological ultrasound or chest radiography). In 4 (1.1\%) cases, ultrasound findings disagreed with CT scans ( 3 false positives and 1 false negative) and thus were considered inaccurate. For the remaining 12 cases, ultrasound images/clips were de-identified and randomised for blinded assessment. Ultrasound findings were confirmed in 10/12 cases (true positives), with one false negative and one false positive (figure 2). Respiratory physician-delivered ultrasound was shown to 
be accurate in 350/356 cases, resulting in an overall accuracy of $98.3 \%(95 \% \mathrm{CI}$ $96.3 \%$ to $99.3 \%)$.

\section{Safety}

The major complication rate was $2 / 235(0.9 \%)$. These procedures were both performed by registrars. Both of these instances were pneumothoraces requiring chest tube insertion. There were no instances of clinically significant intra-pleural haemorrhage requiring intervention, no perforation of viscera and no incidences of pleural infection. Radiological evidence of iatrogenic pneumothorax was reported on post-procedural imaging in 26/235 (11\%). 20 of these instances were registrar performed procedures, and 6 consultant performed. There were four vasovagal episodes and one episode of excessive pain reported. Lastly, 5/235 (2.1\%) interventional scans (4 pleural aspirations, one intercostal drain insertion) failed to obtain pleural fluid, 3 were successfully subsequently aspirated by radiologist, with 2 deemed unsafe.

External assistance was required in 10/357 (2.8\%) cases. Nine patients were referred to radiology for a variety of reasons. Five cases were considered to be unsafe for ward-based procedures by the physician operators, three were referred following failed intervention on the ward, and one case was referred for assistance with diagnostic interpretation of ultrasound findings. One patient was referred directly to cardiothoracic surgery for chest drain insertion in theatre, following a failed 
procedure on the ward. $28 / 35(80 \%)$ of complications occured when procedures were performed by registrars.

\section{$+$ \\ DISCUSSION}

This is the first study to investigate the outcomes of a ward-based thoracic ultrasound service, where examinations are performed by a wide range of operators with varying degrees of experience. The majority of evidence for Respiratory Physician-delivered ultrasound to date has come from studies performed in specialist pleural units by small numbers of highly experienced ultrasound operators ${ }^{6,8}$. In contrast, this study found that over a period of less than three years, 17 individual clinicians, including 9 registrars, conducted ultrasound examinations for the pleural ultrasound service. We believe this to be broadly reflective of Respiratory Medicine in Australian Public Hospitals, where lower patient numbers restrict implementation of highly subspecialised care.

Nine Respiratory Registrars accounted for over $80 \%$ of ultrasound assessments, confirming our assertion that trainee-physicians would be the primary drivers of this service. Respiratory registrars rotate yearly at the RMH, and in some cases may have limited prior experience with thoracic ultrasound. The experience level of Physician operators was not assessed directly in this study, however, operator experience has 
been shown to be associated with significantly lower complication rates ${ }^{9}$. While factors such as limiting the number of authorized proceduralists and ensuring structured training and competency standards can significantly decrease pneumothorax rate, this appears to be independent of whether the operators are senior or trainee physicians ${ }^{10}$. As long as registrars are appropriately trained and have ample hands-on experience ${ }^{11,12}$, we see no intrinsic reason that they should be less proficient than consultants in the use of thoracic ultrasound.

Our findings confirm high accuracy of bedside thoracic ultrasound for detection of pleural fluid, with $98.3 \%(95 \% \mathrm{CI}=96.3 \%$ to $99.3 \%)$ of assessments shown to be correct. This value is consistent with others reported within the literature ${ }^{13}$, most significantly the study by Rahman ${ }^{6}$.

Overall, there were six cases in which the physician-delivered ultrasound was found to be inaccurate. As $\mathrm{CT}$ is regarded as the gold standard for the detection of pleural effusions ${ }^{14-16}$, instances in which there was disagreement between $\mathrm{CT}$ and ultrasound findings, the CT findings were considered correct. There was one case in which a moderate effusion was noted on CT that was not identified on ultrasound, however the physician-operator did acknowledge at the time that the views were inadequate, offering to review at a later date if required. In three further cases an effusion was reported during the ultrasound assessment that was not seen on concurrent CT scans. Interestingly, in each of these three cases, blinded review of the ultrasound findings by two Respiratory Physicians and an experienced thoracic Radiologist revealed an 
apparent trivial effusion. While these cases have been recorded as false positives for the purposes of this study, it does raise interesting questions about the relative sensitivity of these imaging techniques. While CT is generally regarded as the gold standard, an expert panel reviewing the literature of point-of-care ultrasound found Level-A evidence that ultrasound was as accurate as CT in detecting effusions ${ }^{1}$. Additionally, ultrasound has been shown to detect effusions as small as $3-5 \mathrm{~mL}^{14}$. It is possible that trivial, gravity-dependent effusions may be easier to visualise on ultrasound assessment of an upright patient than on supine CT, where small volume effusions may spread out to form a pleural film. Regardless, in each case the effusions were deemed to be insufficient for intervention by the physician-operator, and thus are unlikely to have negatively impacted on patient outcomes.

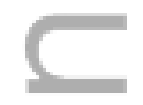

This study yielded a major complication rate of $0.9 \%$. While there is no accepted standard within the literature, this value is comparable to two seminal prospective studies of respiratory physician-delivered thoracic ultrasound by Rahman and Ault ${ }^{6,8}$, which reported rates of $0.5 \%$ and $0.98 \%$ respectively. Using these studies as a reference standard, it would appear that procedures performed by our ultrasound service are acceptably safe. We believe this to be particularly significant, given both of the aforementioned studies were carried out by highly experienced physicianoperators.

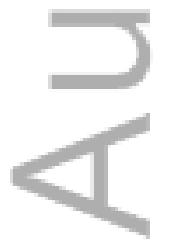


Post-procedural imaging revealed evidence of pleural air-collections in $11.0 \%$ of interventional scans in this study, exceeding pneumothorax rates reported within the recent literature. Despite this, we do not believe that ultrasound guided procedures performed by this unit were associated with a high degree of morbidity. Overall there were two instances of pneumothorax requiring intervention $(0.9 \%$ of interventional scans). These results compare favourably with meta-analysis of the literature, which found $1.7 \%$ of all thoracentesis require chest tube insertion to evacuate symptomatic pneumothoraces ${ }^{9}$. The remaining cases were clinically insignificant and did not require further intervention. As they did not significantly affect outcomes for patients they were considered to be minor complications. Post-thoracentesis chest radiography was not routinely performed for small volume aspirations, but was performed in symptomatic patients and post large volume aspiration and chest tube insertions. While small pneumothoraces may have been missed, these were unlikely to be of clinical significance.

A number of mechanisms are recognised for the development of pneumothorax following thoracentesis or intercostal catheter insertion. Air enters the pleural space via penetrating lung injury, during the procedure or rarely through the resultant wound. An under-recognised cause of air within the pleural space is ex-vacuo pneumothorax or 'trapped lung, ${ }^{17}$. This phenomenon is commonly encountered, particularly in chronic effusions, where the lung has become tethered and thus cannot re-expand to confirm to the shape of the thoracic cavity as fluid is removed. It is 
thought that transient parenchymal-pleural fistulae develop due to stress on the visceral pleura during aspiration, allowing air to enter the pleural space. Trapped lung is an unavoidable consequence of thoracentesis in these cases and has been shown to account for the vast majority of cases of unintentional pneumothorax ${ }^{18}$.

The higher rate of pneumothorax in procedures performed by registrars again suggests that complication rates are higher when procedures are performed by less experienced clinicians, and that ultrasound guided pleural procedures should be performed by appropriately trained and experienced operators, or where appropriate supervision is available.

External input was required in $2.8 \%$ of cases, which was slightly lower than the $4.0 \%$ reported by Rahman ${ }^{6}$. This is perhaps surprising given the relative inexperience of our operators. The higher rate of pneumothoraces demonstrated in our series, as well as the four failed procedures, suggests that a lower threshold for seeking input from radiology or more senior colleagues is of utmost importance to avoid complications. Assistance was sought once for diagnostic interpretation, indicating that operators are generally confident in their ability to interpret ultrasound images.

There are a number of limitations to this study. Firstly, being a retrospective study we were reliant on complete collection of data at the time of the procedures. As data for major complications was followed up using objective radiology and pathology records, we believe these values to be accurate. Pleural manometry was not routinely 
performed in this series but would aid in the differentiation of trapped lung and pneumothorax.

Despite clear evidence for ultrasound guidance in pleural procedures, up to one third of respiratory physicians are yet to adopt this practice ${ }^{3,7}$. In our region, a number of barriers have been identified including lack of access to equipment and inadequate training and expertise, despite there are currently existing sufficient capacity to deliveraccredited training for respiratory physicians in Australia and New Zealand ${ }^{7}$. Furthermore, the purchase of bedside ultrasound equipment has been shown to be generally cost-beneficial due to reductions in hospital stay resulting from complications and delays in radiology ${ }^{19,20}$. The results of our study add to the evidence for respiratory physician-delivered thoracic ultrasound ${ }^{5}$.

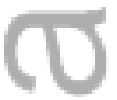

In conclusion, a ward-based clinician-delivered thoracic ultrasound service appears to be safe and accurate in the diagnosis and management of pleural effusions. This is the first study to assess the outcomes associated with such a service where trainee-physicians perform the majority of ultrasound examinations. Our data supports the assertion that close liaison with radiology may ensure safety and efficacy of such a service. The results of this study suggest that Respiratory Physician-delivered thoracic ultrasound is feasible Australian public hospitals. 


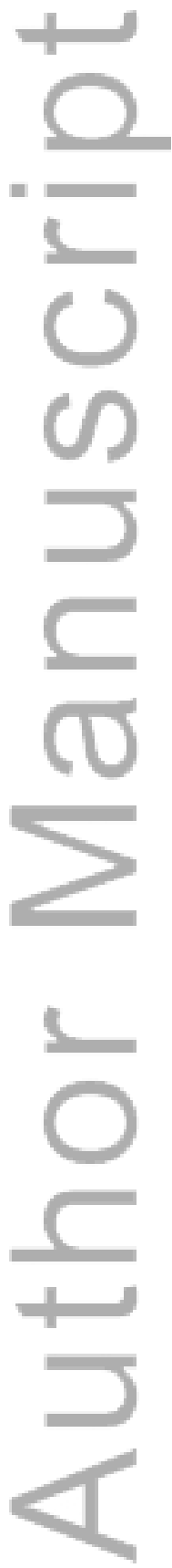

This article is protected by copyright. All rights reserved. 


\section{REFERENCES}

1 Volpicelli G, Elbarbary M, Blaivas M, Lichtenstein DA, Mathis G, Kirkpatrick AW, Melniker L, Gargani L, Noble VE, Via G, Dean A, Tsung JW, Soldati G, Copetti R, Bouhemad B, Reissig A, Agricola E, Rouby JJ, Arbelot C, Liteplo A, Sargsyan A, Silva F, Hoppmann R, Breitkreutz R, Seibel A, Neri L, Storti E, Petrovic T, International Liaison Committee on Lung Ultrasound for International Consensus Conference on Lung U. International evidence-based recommendations for point-of-care lung ultrasound. Intensive care medicine. 2012; 38: 577-91.

2 Koegelenberg CF, von Groote-Bidlingmaier F, Bolliger CT. Transthoracic ultrasonography for the respiratory physician. Respiration. 2012; 84: 337-50. 3 Hooper CE, Welham SA, Maskell NA, British Thoracic S. Pleural procedures and patient safety: a national BTS audit of practice. Thorax. 2015; 70: 189-91.

4 Havelock T, Teoh R, Laws D, Gleeson F, Group BTSPDG. Pleural procedures and thoracic ultrasound: British Thoracic Society Pleural Disease Guideline 2010. Thorax. 2010; 65 Suppl 2: ii61-76.

5 Hew M, Heinze S. Chest ultrasound in practice: a review of utility in the clinical setting. Internal medicine journal. 2012; 42: 856-65.

6 Rahman NM, Singanayagam A, Davies HE, Wrightson JM, Mishra EK, Lee YC, Benamore R, Davies RJ, Gleeson FV. Diagnostic accuracy, safety and utilisation of respiratory physician-delivered thoracic ultrasound. Thorax. 2010; 65: 449-53.

7 Hannan LM, Steinfort DP, Irving LB, Hew M. Direct ultrasound localisation for pleural aspiration: translating evidence into action. Internal medicine journal. 2014; 44: 50-6.

8 Ault MJ, Rosen BT, Scher J, Feinglass J, Barsuk JH. Thoracentesis outcomes: a 12-year experience. Thorax. 2015; 70: 127-32.

9 Gordon CE, Feller-Kopman D, Balk EM, Smetana GW. Pneumothorax following thoracentesis: a systematic review and meta-analysis. Archives of internal medicine. 2010; 170: 332-9.

10 Duncan DR, Morgenthaler TI, Ryu JH, Daniels CE. Reducing iatrogenic risk in thoracentesis: establishing best practice via experiential training in a zero-risk environment. Chest. 2009; 135: 1315-20.

11 Moy RJ, Chapman AL, Bapusamy A. The effectiveness of an informal teaching programme for junior doctors identifying pleural effusions using ultrasound at the bedside. Journal of the Royal Army Medical Corps. 2010; 156: 233-5.

12 Wong CA, Lee O, Kennedy Y, Kenealy H, Hood C, Sivakumaran P, Lee YC. The training, experience, and confidence of junior doctors in performing pleural procedures. N Z Med J. 2009; 122: 23-32. 
13 Grimberg A, Shigueoka DC, Atallah AN, Ajzen S, Iared W. Diagnostic accuracy of sonography for pleural effusion: systematic review. Sao Paulo Med J. 2010; 128: 90-5.

14 Gryminski J, Krakowka P, Lypacewicz G. The diagnosis of pleural effusion by ultrasonic and radiologic techniques. Chest. 1976; 70: 33-7.

15 Yu CJ, Yang PC, Wu HD, Chang DB, Kuo SH, Luh KT. Ultrasound study in unilateral hemithorax opacification. Image comparison with computed tomography. The American review of respiratory disease. 1993; 147: 430-4. 16 Lichtenstein D, Goldstein I, Mourgeon E, Cluzel P, Grenier P, Rouby JJ. Comparative diagnostic performances of auscultation, chest radiography, and lung ultrasonography in acute respiratory distress syndrome. Anesthesiology. 2004; 100: 9-15.

17 Boland GW, Gazelle GS, Girard MJ, Mueller PR. Asymptomatic hydropneumothorax after therapeutic thoracentesis for malignant pleural effusions. AJR American journal of roentgenology. 1998; 170: 943-6.

18 Heidecker J, Huggins JT, Sahn SA, Doelken P. Pathophysiology of pneumothorax following ultrasound-guided thoracentesis. Chest. 2006; 130: 1173-84.

19 Patel PA, Ernst FR, Gunnarsson CL. Ultrasonography guidance reduces complications and costs associated with thoracentesis procedures. J Clin Ultrasound. 2012; 40: 135-41.

20 Bateman K, Downey DG, Teare T. Thoracic ultrasound for pleural effusion: delays and cost associated with departmental scanning. Respir Med. 2010; 104: 612-4.
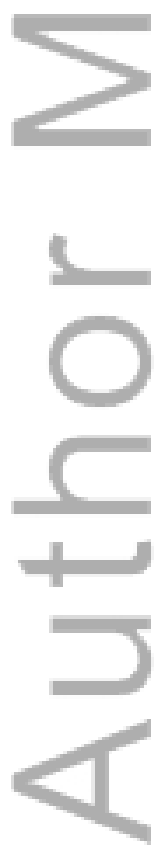

This article is protected by copyright. All rights reserved. 
TABLE 1: Criteria for diagnostic accuracy of ultrasound assessments.

\section{CRITERIA FOR DIAGNOSTIC ACCURACY}

1. Pleural fluid successfully obtained at the time of, or within seven days of the

initial ultrasound assessment.

2. Confirmation of ultrasound findings by other imaging studies (CT, MRI, ultrasound) performed within seven days of the ultrasound assessment as part of routine clinical care and reported by a radiologist.

3. Confirmation of ultrasound findings by chest radiography performed within seven days of the ultrasound assessment as part of routine clinical care and reported by a radiologist.

4. In the absence of any of the above, randomised and anonymised ultrasound images or videos were reviewed by two consult respiratory physicians blind to the findings of the initial ultrasound assessment, to determine the presence or absence of a pleural effusion.

5. In case where there was disagreement, the randomised and anonymised ultrasound images or videos were reviewed by a consultant thoracic radiologist ) 


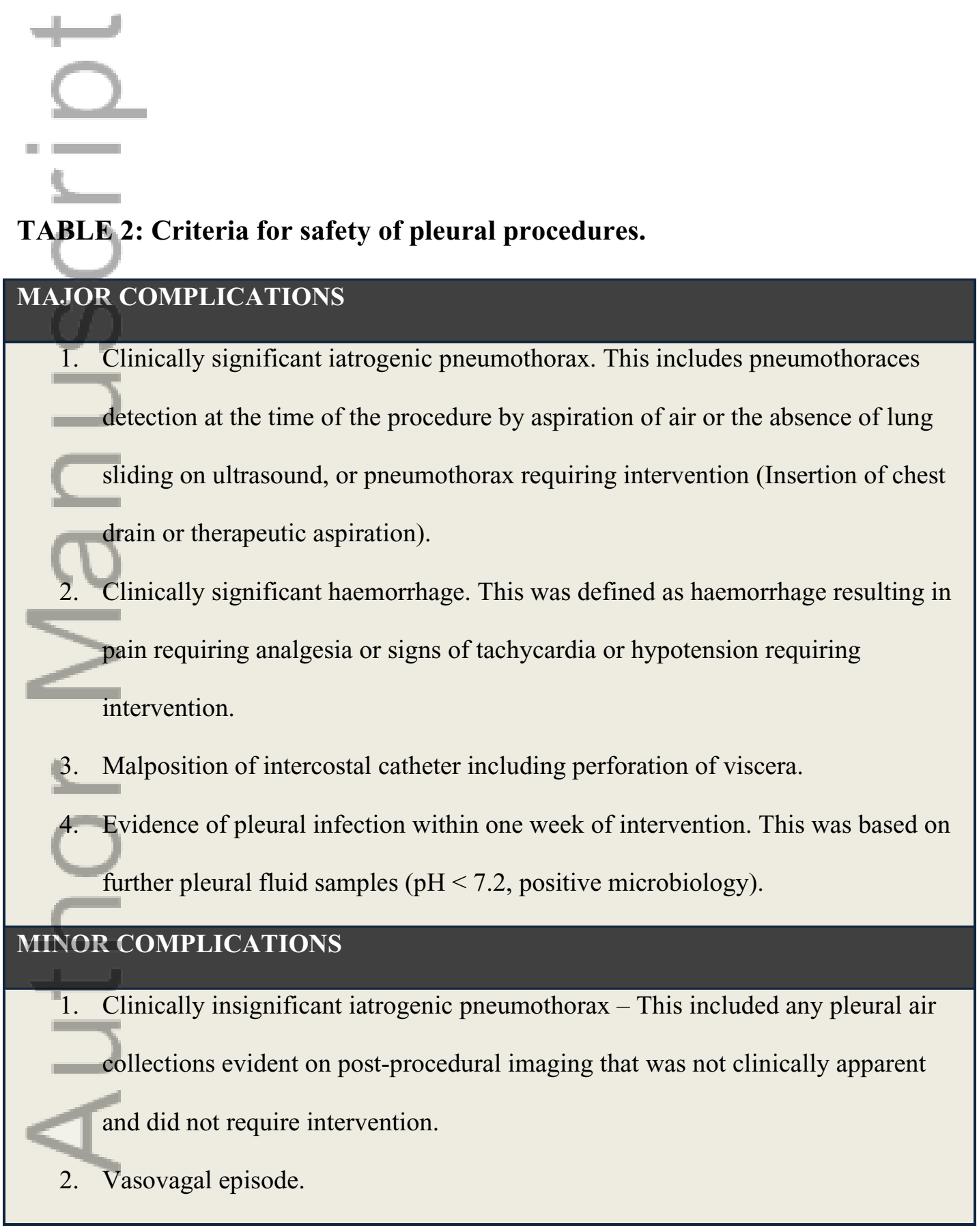

This article is protected by copyright. All rights reserved. 
3. Failure to obtain pleural fluid during procedure.

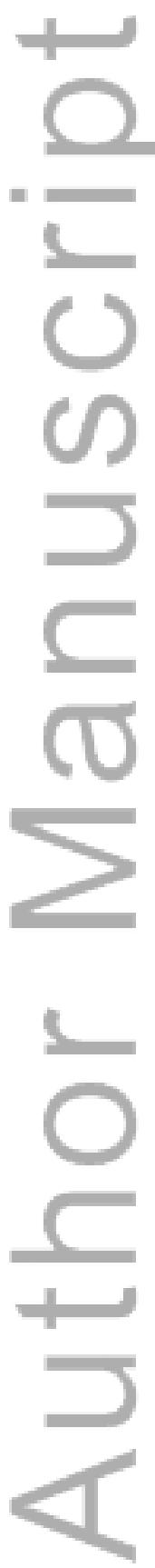

This article is protected by copyright. All rights reserved. 
Figure legends

Figure 1-Flowchart depicting the diagnostic accuracy by successfully aspirating pleural fluid and diagnostic ultrasound.

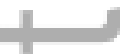

Figure 2-Flowchart depicting the diagnostic accuracy requiring radiology confirmation
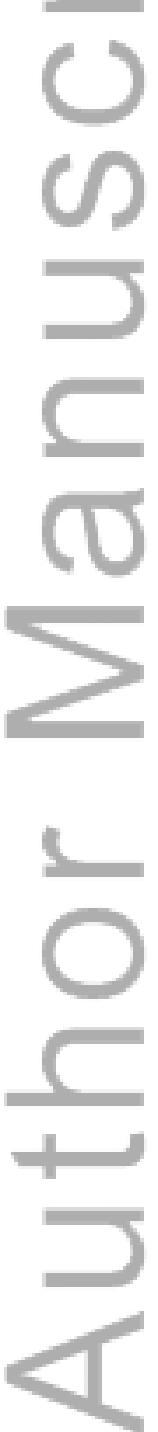

This article is protected by copyright. All rights reserved. 


\section{University Library}

\section{- M M N E R VA A gateway to Melbourne's research publications}

Minerva Access is the Institutional Repository of The University of Melbourne

Author/s:

Hammerschlag, G;Denton, M;Wallbridge, P;Irving, L;Hew, M;Steinfort, D

Title:

Accuracy and safety of ward based pleural ultrasound in the Australian healthcare system

Date:

2017-04-01

Citation:

Hammerschlag, G., Denton, M., Wallbridge, P., Irving, L., Hew, M. \& Steinfort, D. (2017).

Accuracy and safety of ward based pleural ultrasound in the Australian healthcare system. RESPIROLOGY, 22 (3), pp.508-512. https://doi.org/10.1111/resp.12932.

Persistent Link:

http://hdl.handle.net/11343/292107 